\title{
Proximal Femoral Allograft for Major Segmental Femoral Bone Loss: A Systematic Literature Review
}

\author{
B. A. Rogers, A. Sternheim, D. Backstein, O. Safir, and A. E. Gross \\ Department of Orthopaedic Surgery, Mount Sinai Hospital, Toronto, ON, Canada M5G 1X5 \\ Correspondence should be addressed to B. A. Rogers, benedictrogers@hotmail.com \\ Received 27 March 2011; Revised 16 June 2011; Accepted 7 July 2011 \\ Academic Editor: Robert Michael Meneghini
}

Copyright () 2011 B. A. Rogers et al. This is an open access article distributed under the Creative Commons Attribution License, which permits unrestricted use, distribution, and reproduction in any medium, provided the original work is properly cited.

As the indications for total hip arthroplasty increase, the prevalence of extensive proximal femoral bone loss will increase as a consequence of massive osteolysis, stress shielding and multiple revisions. Proximal femoral bone stock deficiency provides a major challenge for revision hip arthroplasty and is likely to account for a significant future caseload. Various surgical techniques have been advocated included impaction allografting, distal press-fit fixation and massive endoprosthetic reconstruction. This review article provides a systematic review of the current literature to assess the outcome of revision hip arthroplasty using allograft to reconstruction massive proximal femoral bone loss.

\section{Introduction}

As the need for total hip arthroplasty increases, the incidence of extensive proximal femoral bone loss will increase as a consequence of massive osteolysis, stress shielding and multiple revisions [1-5]. Proximal femoral bone stock deficiency provides a major challenge for revision hip arthroplasty and is likely to account for a significant future caseload [6].

Various surgical techniques have been advocated included impaction allografting techniques $[7,8]$, distal press-fit fixation $[9,10]$, and massive endoprosthetic reconstruction [11-13]. Individual studies have reported a 58\% to $84 \%$ survivorship of massive endoprosthetic reconstruction (or megaprostheses) with average followup ranging from 5 to 10 years [11-13]. A recent retrospective review of 403 proximal femoral replacements (endoprosthetic reconstructions) from five institutions reported a 10- and 15-year survival rate of $75 \%$, with mechanical causes being the commonest mode of failure [14].

A proximal femoral allograft reconstruction requires the use of a prosthesis bridging the host-allograft junction and obtaining fixation in the distal femur. The enhancement of future bone stock is an important advantage purported to this method of reconstruction that has been utilized in proximal femoral bone loss secondary to tumors and aseptic osteolysis. Differences in the morphology of the hostallograft junction, the use of cement, and the method of attachment of the host abductor musculature have all been described. follows:

The three principal aims of this systematic review were as

(1) to document variations in the surgical techniques used,

(2) to assess the clinical outcome of allograft prosthesis composites (APC) for massive proximal femoral bone loss,

(3) to quantify complication rates in relation to the surgical technique used.

\section{Methods}

A comprehensive search of the MEDLINE, EMBASE, and the National Institutes of Health online database PubMed from the earliest records to the time of review (January 2011) was performed. The following Medical Subject Headings (MeSH) terms were used: "allograft," "composite graft" in the manuscript title, and "proximal femoral" in the manuscript abstract. The keywords were used as both text words and Medical Search Headings (MeSH terms). 
Two authors (B. A. Rogers, A. Sternheim) independently applied the search strategy to the different databases and reviewed the selected references. Titles, abstracts and papers were reviewed independently.

The following inclusion criteria were used:

(1) studies retrieved by the database search using the Medical Subject Headings detailed above,

(2) studies specifically reporting outcomes relating to proximal femur composite.

The following exclusion criteria were used:

(1) non-English language,

(2) case reports,

(3) review articles,

(4) not relating to human surgery,

(5) patients with advanced oncological pathology,

(6) followup less than 2 years.

Where more than one publication existed relating to the outcomes of same cohort of patients from the same institution, the most recent publication only was used.

Full-text manuscripts were obtained and reviewed for the studies identified using the above criteria. The method of review followed the authoritative methodology described by Mohit [15].

Allograft-prosthetic composite (APC) is a technique used to restore bone stock and mechanical stability to the proximal femur (see Figures 1(a)-1(d), and 2). The studies analyzed in this literature review consider a single technique, APC, rather than a single diagnosis; this technique has been utilized for oncological and nononcological surgery.

Eight studies report on APC used in non-oncological conditions (septic or aseptic loosening) and six report on surgeries performed for malignant or nonmalignant proximal femoral pathology. Two studies report on patient cohorts with both indications.

The primary outcome of interest was further revision of the femoral component and the secondary outcomes of interest were other complications such as infection, dislocation, and nonunion.

Statistical analysis was performed on the selected papers to assess the pooled success rate. The effect (proportion) was calculated for every individual study and the pooled effect considering all the studies.

\section{Results}

3.1. Studies. Sixteen studies reported on outcomes of proximal femoral composite allograft used to reconstruct major bone defects (see Table 1). All studies were retrospective case series and provide level IV evidence. All studies were published within the last fifteen years. Average followup ranged from 2 to 16.2 years. The total number of allograft reconstructions reported in all the studies was 498. The surgical techniques, clinical outcomes and complications were collated for all these published studies.
3.2. Surgical Techniques. The described surgical techniques varied, as shown in Tables 2 and 3.

Four studies described the complete resection of the proximal femur as the approach employed; however, the transtrochanteric approach was the most common reported.

Regarding the morphology of the osteotomy used at the junction between the proximal allograft and distal host femur, 8 studies reported a transverse femoral osteotomy, 3 that were augmented with plate fixation to enhance stability at the allograft-host junction. The remainder of the studies reported either a step or oblique femoral osteotomy.

The management of the proximal host femur varied. In 9 studies the proximal host femur was fully resected, with 5 studies using the split host proximal femur as an onlay graft after the APC had been inserted. Two studies did not detail this aspect of the surgical technique. Four studies reported the use of cortical strut allografts to reinforce the allografthost junction $[6,18,22,29]$, with one study reporting use in every case [22].

The techniques used for fixation of the prosthesis to the allograft, and for distal fixation to the host femur is shown in Table 3. There are 14 studies reporting cemented fixation of the prosthesis to the allograft; however, distal fixation varied with 6 uncemented, 4 cemented, 5 studies employed a variety of techniques and one study did not report.

3.3. Clinical Outcomes. The primary outcome of interest was further revision of the femoral component, and Table 4 shows the reported failure rate and success rate for the allograft prosthesis composite in each study. The success rate was defined as the reported survivorship of the APC.

The total cohort included 498 patients with a mean follow up of 8.1 years (range 2 to 16.2 years). The pooled success rate was $81 \%$ (95\% CI 77\%-86\%).

However, the number of cases and length of followup varied substantially between the studies. For example, Roque et al. reported an $82 \%$ survivorship rate for 73 allograft prosthesis reconstructions at 6.7 years followup [23], whereas Safir et al. reported 15 year Kaplan-Meier survivorship data on 50 patients of $82 \%$ [19].

3.4. Complications. Table 4 details the reported major complications. The infection rate ranged from $0 \%$ to over $21 \%$, with a pooled mean of $8 \%$. The two studies with a reported infection rate of over $20 \%$ had only 14 and 15 patients, respectively $[13,29]$. Conversely, the four studies reporting the lowest infection rates ( 0 to $4 \%$ ) had a mean patient cohort of 40 patients $[6,16,17,20]$.

Dislocation is a significant postoperative complication, however five out of the sixteen studies did not report the incidence of dislocation $[13,24,25,27,28]$. For the eleven studies that did report dislocation rate the mean was $12.8 \%$ with a range $0 \%[16,23]$ to $40 \%[18]$. The mean reported dislocation rate in studies that used a technique of splitting the host proximal femur to use as an onlay graft was $9.8 \%$, compared to $14.9 \%$ in studies that resected the entire proximal femur. 


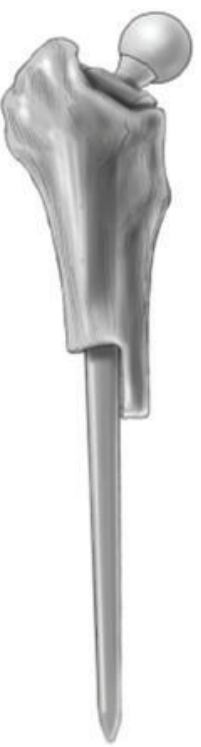

(a)

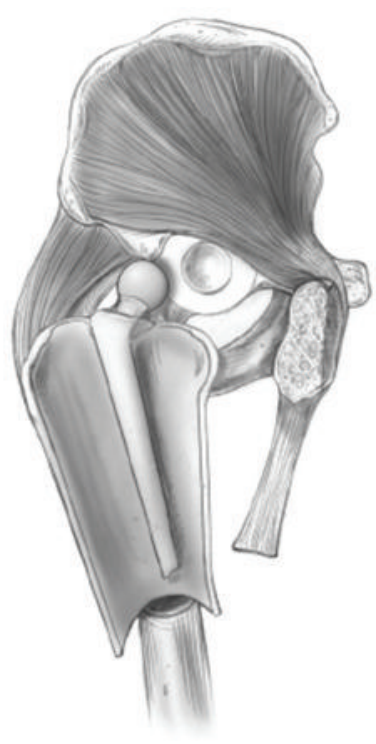

(b)

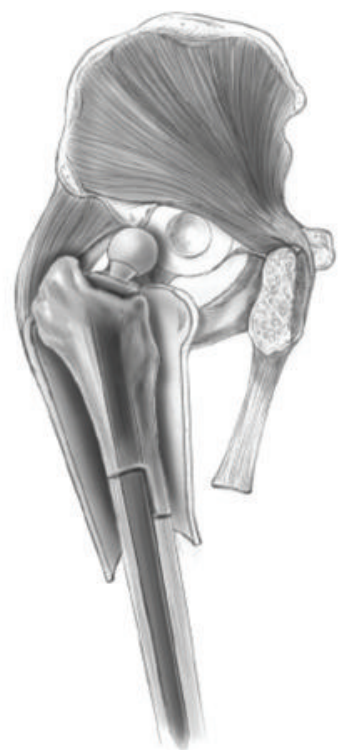

(c)

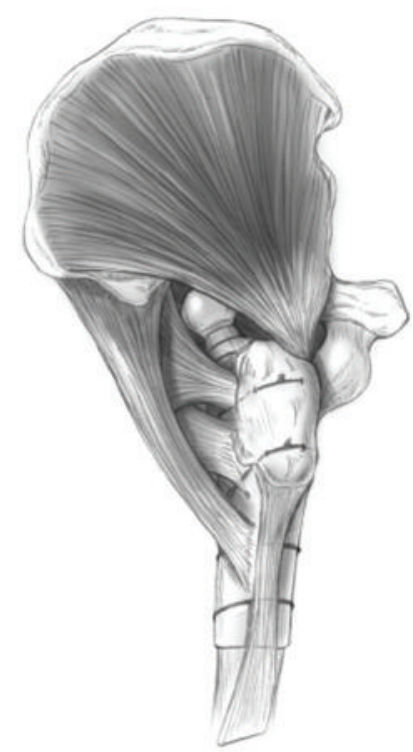

(d)

FIgUre 1: (a) Prosthesis cemented into allograft. (b) Trochanteric slide approach to hip, with lateral cortex osteotomy to facilitate removal of in situ femoral component. (c) Allograft-prosthesis composite inserted into host, with junctional step cut. (d) Remnants of host proximal femur are fixed around allograft, especially at the allograft-host junction, and the greater trochanter reattached.

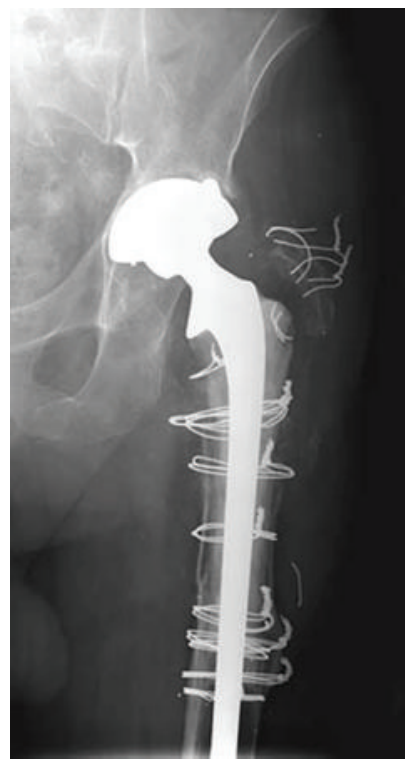

Figure 2: A radiograph 17 years after proximal femoral allograft (reprinted from Safir et al. [19]).

Failure of the APC, either resulting from aseptic loosening or fracture (Table 4) ranges from $0 \%$ to $28 \%$. The mean reported aseptic loosening or fracture rate was $13.7 \%$ for studies that used cement for fixation into distal host femur, compared to $9.1 \%$ for those studies using uncemented fixation in the distal host femur. However, the difference was not statistically different.

\section{Discussion}

4.1. Clinical Outcome. Severe proximal femoral bone loss is creating an increasing caseload of complex cases for the reconstructive hip surgeon [6]. The use of allograft prosthesis composite (APC) is one surgical solution used to address this problem and restore mechanical stability to the proximal femur. This analysis reviews the surgical techniques, clinical outcomes and complication, incorporating a total patient cohort of 498 from sixteen studies with a mean follow up of 8.1 years (range 2 to 16.2 years). The pooled success rate was $81 \%$ (95\% CI 77\%-86\%), see Table 4, and provides evidence that this technique is valid and durable when performed by suitable trained and experienced surgeons, in institutions with the facilities to support such complex surgery.

4.2. Surgical Approaches and Complications. Surgical technique varied between the studies with regard to surgical approach, storage technique of the allograft bone, fixation techniques of the prosthesis to the proximal allograft, distal host femur and the junction between the allograft and host bone (see Table 2).

Several different surgical approaches were utilized in the reported studies. Four studies all pertain to tumour resection used a direct lateral approach with complete resection of the proximal femur. Trochanteric slide osteotomy was used in two studies both reported on patients who had revision of a failed hip arthroplasty. A transtrochanteric approach was reported by Vastel et al. and led to a high rate of trochanteric nonunion (25/34) with the authors recommending the use of a trochanteric plate to avoid proximal migration of the trochanter [20]. 
TABLE 1: Sixteen studies using allograft prosthetic composite in the treatment of proximal femoral bone loss, number of patients per study, primary diagnosis, and mean followup.

\begin{tabular}{lcccc}
\hline & Study & $n$ & Primary diagnosis & Mean followup (yrs) \\
\hline 1 & Chandler et al. [6] & 30 & Aseptic & 2 \\
2 & Langlais et al. [16] & 21 & Tumor & 6 \\
3 & Haddad et al. [17] & 55 & Tumor, Aseptic, Septic revision & Tumor \\
4 & Zehr et al. [13] & 14 & Aseptic failure & 10 \\
5 & Zmolek and Dorr [18] & 15 & Septic, aseptic & 16.2 \\
6 & Safir et al. [19] & 50 & Aseptic failure & 7.1 \\
7 & Vastel et al. [20] & 44 & Aseptic & 12 \\
8 & Babis et al. [21] & 72 & Aseptic, septic loosening & 4.2 \\
9 & Lee et al. [22] & 15 & Tumor & Tumor \\
10 & Roque et al. [23] & 73 & Tumor & 6.7 \\
11 & Biau et al. [24] & 32 & Tumor & 5.6 \\
12 & Donati et al. [25] & 22 & Aseptic, septic loosening & 4.8 \\
13 & Farid et al. [26] & 20 & Tumor & 6.3 \\
14 & Graham and Stockley [27] & 25 & Aseptic, septic loosening & 7.5 \\
15 & Muscolo et al. [28] & 37 & 15 & 7.6 \\
\hline
\end{tabular}

TABLE 2: Surgical techniques used including approach, the type of femoral osteotomy performed at the host bone-allograft junction, and whether the host proximal femur was resected or split and used as an onlay graft. NR: not reported. Study numbers correlate with Table 1.

\begin{tabular}{|c|c|c|c|c|}
\hline Study & $n$ & Surgical approach & Femoral osteotomy & Host proximal femur \\
\hline 1 & 30 & Trochanteric slide & Step cut (7) transverse (23) & NR \\
\hline 2 & 21 & Complete resection & Step cut & Resected \\
\hline 3 & 55 & NR & Transverse (28), step cut (12) & Resected \\
\hline 4 & 14 & Complete resection & NR & Resected \\
\hline 5 & 15 & Posterolateral (9), trochanteric Slide (2) & Oblique & Resected \\
\hline 6 & 50 & Trochanteric slide & Step cut & Split and onlay \\
\hline 7 & 44 & Transtrochanteric & Transverse & Split and onlay \\
\hline 8 & 72 & Hardinge (44), posterior (11), transtrochanteric (17) & Step cut (62), telescoping (10) & Split and onlay \\
\hline 9 & 15 & Transtrochanteric & Transverse (9), step cut (6) & NR \\
\hline 10 & 73 & Complete resection & NR & Resected \\
\hline 11 & 32 & Trochanteric slide (12), resection (20) & Transverse & Resected \\
\hline 12 & 22 & Complete resection & Transverse & Resected \\
\hline 13 & 20 & NR & NR & Resected \\
\hline 14 & 25 & Trochanteric slide & Step cut & Split and onlay \\
\hline 15 & 37 & Posterolateral (28), transtrochanteric (10) & Transverse & Resected \\
\hline 16 & 15 & Transtrochanteric & Transverse & Split and onlay \\
\hline
\end{tabular}

Trochanteric nonunion and abductor strength are also influenced by surgical approach. The trochanteric slide osteotomy aims to maintain the continuum of tissue from the abductors and the greater trochanter to the vastus lateralis. This approach has been reported to have a higher rate of trochanteric union [30]. The trochanteric slide osteotomy has been further modified to maintain the external rotators and thus improve hip stability [30-32].

The junctional osteotomy between the host femur and the proximal allograft was transverse, oblique or step-cut (see Table 2). A step-cut osteotomy may offer more rotational stability while an oblique osteotomy may offer more surface area for bone in-growth compared to a transverse osteotomy. Langlais et al. reported on two cases of loosening with junctional failure that they attributed to a lack of a stepcut osteotomy at the junction [16]. This junction may be further reinforced with strut allografts $[6,18,22,29]$. Nonunion of the junction between the native femur and the proximal allograft causes macro motion at the junction that is treated with bone grafting, plating, and/or a strut allograft [16]. Host-allograft junctional nonunion may be reduced by augmentation with additional autologous bone graft and supporting it with either a plate or a strut allograft. Several studies highlight the bone union at the host-allograft 
TABLE 3: Table showing methods of implantation of prosthesis into allograft to form the allograft prosthesis composite (APC) and methods for securing APC to distal host femur. NR: not reported. Study numbers correlate with Table 1.

\begin{tabular}{lrr}
\hline Study & Allograft-prosthesis fixation & APC-host bone fixation \\
\hline 1 & Cemented & Uncemented \\
2 & Cemented & Cemented \\
3 & Cemented & Cemented \\
4 & Cemented (16), uncemented (2) & Cemented(14), Uncemented(2), plating(2) \\
5 & Uncemented & Uncemented + plating \\
6 & Cemented & Uncemented \\
7 & Cemented & Cemented \\
8 & Cemented & Uncemented (44), cemented (22) \\
9 & Cemented & Uncemented (12), cemented (3) \\
10 & NR & NR \\
11 & Cemented & Cemented \\
12 & Cemented & Uncemented \\
13 & Cemented & Varied \\
14 & Cemented & Uncemented \\
15 & Cemented & Uncemented + plating \\
16 & Cemented & Uncemented (13), cemented (2), plating \\
\hline
\end{tabular}

TABLE 4: Table showing complications of prosthesis into allograft to form the allograft prosthesis composite (APC) and methods for securing APC to distal host femur. The total cohort included 498 patients with a mean follow up of 8.1 years (range 2 to 16.2 years). The pooled success rate was 81\% (95\% CI 77\%-86\%). Success rate: APC not revised. NR: not reported. Study numbers correlate with Table 1.

\begin{tabular}{|c|c|c|c|c|c|c|}
\hline Study & $n$ & Failed constructs & Success rate & Infection & Dislocation & Aseptic loosening or fracture \\
\hline 1 & 30 & 3 & $90 \%$ & $1(3.3 \%)$ & $5(16.7 \%)$ & $4(13.3 \%)$ \\
\hline 2 & 21 & 2 & $82 \%$ & 0 & 0 & $6(28.6 \%)$ \\
\hline 3 & 55 & 6 & $85 \%$ & $2(3.6 \%)$ & $4(7.3 \%)$ & $5(9.1 \%)$ \\
\hline 4 & 14 & 4 & $78 \%$ & $3(21.4 \%)$ & NR & $1(7.1 \%)$ \\
\hline 5 & 15 & 3 & $73 \%$ & $1(6.7 \%)$ & $6(40 \%)$ & $2(13.3 \%)$ \\
\hline 6 & 50 & 8 & $84 \%$ & $2(4 \%)$ & $4(8 \%)$ & $7(14.0 \%)$ \\
\hline 7 & 44 & 4 & $91 \%$ & $1(2.3 \%)$ & $6(13.6 \%)$ & $3(6.8 \%)$ \\
\hline 8 & 72 & 19 & $66 \%$ & $5(6.9 \%)$ & $8(11.1)$ & $\begin{array}{l}14 \text { Loosening (4), resorption (3), nonunion (2), } \\
\text { fracture (4), stem fracture (1) }\end{array}$ \\
\hline 9 & 15 & 2 & $87 \%$ & $1(6.7 \%)$ & $1(6.7 \%)$ & $1(6.7 \%)$ \\
\hline 10 & 73 & 13 & $82 \%$ & $8(10.9 \%)$ & 0 & $11(15.1 \%)$ \\
\hline 11 & 32 & 9 & $72 \%$ & $4(12.5 \%)$ & NR & $5(15.6 \%)$ \\
\hline 12 & 22 & 2 & $91 \%$ & $1(4.5 \%)$ & NR & $1(4.6 \%)$ \\
\hline 13 & 20 & 1 & $95 \%$ & $1(5.0 \%)$ & $2(10 \%)$ & 0 \\
\hline 14 & 25 & 2 & $92 \%$ & $1(4.0 \%)$ & NR & $2(8.0 \%)$ \\
\hline 15 & 37 & 10 & $73 \%$ & $3(8.1 \%)$ & NR & $7(18.9 \%)$ \\
\hline 16 & 15 & 5 & $67 \%$ & $3(20 \%)$ & $1(6.7)$ & $4(20.7 \%)$ \\
\hline
\end{tabular}

junction as a key factor in achieving stability of the composite graft, and thereby lowering the chance of mechanical failure $[17,19,21,33]$.

Cement fixation of the prosthesis to the allograft with cementless fixation to the host femur was used in seven studies (see Table 3) [6, 19, 24, 25, 27-29]. The rationale for cement fixation in the allograft-prosthesis composite is that in-growth and on-growth would not be expected at the allograft prosthesis interface. Only Zmolek and Dorr reported a fully uncemented fixation of the prosthesis and allograft in 11 patients with similar rates of success compared to other studies [18].

Regarding distal fixation to the host femur, an uncemented technique was principally employed in nine studies $[6,18,19,22,24,25,27-29]$, cemented in four studies $[13,16,17,20]$, mixed cemented and uncemented distal fixation in one study [21], and one study did not report whether or cement was used (see Table 3 ). 
For the studies that utilized cementless distal fixation, some employed a press-fit or interference technique whereas others used an oblique or step-cut junctional osteotomy. Safir et al. used an uncemented technique in the distal host femur with a step-cut or oblique osteotomy affording direct loading at the allograft-host femur junction [19]. The authors support the concept that direct loading of the hostallograft junction minimizes allograft resorption. The distal femur being initially reamed to the optimal size, with the proximal femoral allograft also reamed and broached until a good fit was achieved for the long-stem femoral prosthesis. The mismatch in the medullary sizes of the host bone and the allograft resulted in a good press-fit fixation never being achieved between the femoral stem and the distal host femur. Further, the allograft was never over reamed to accommodate a larger femoral component for the host femur. In contrast, Haddad et al. cemented the prosthesis to the distal femur thus stress shielding, the allograft and commented that this may explain the high rate of graft resorption (17\%) observed [17].

Overall, cemented fixation in the distal host bone was associated with a higher rate of aseptic loosening or fracture $(13.7 \%)$ when compared to uncemented distal fixation (9.1\%; see Table 4). Whilst the difference was not statistically significant, the benefit of uncemented distal fixation is the reduced risk of junctional nonunion between the host femur and allograft.

The population cohorts, the duration, and complexity of the surgery result in infection rates for APC being greater than that for primary hip arthroplasty (see Table 4). Considering these factors, the pooled $8 \%$ infection rate is not unacceptable. The infection rate is; however, related to quantity performed with the lowest infection rates (0 to $4 \%$ ) being reported in those studies with the greater number of cases $[6,16,17,20]$. Although observer bias may influence this data, a greater caseload and experience is likely to be beneficial.

The use of native proximal femur with its soft-tissue attachments as an onlay graft around the composite allograft was reported in five studies [19-21, 27, 29]. This vascularised viable bone can promote in-growth into the allograft and preserves the abductor mechanism and short external rotators. These five studies report a lower mean dislocation rate of $9.8 \%$, compared to $14.9 \%$ (see Table 4 ). From the surgical approaches detailed in these studies, the risk of dislocation may be minimized by:

(1) preservation of the host posterior capsular structures if possible,

(2) good biomechanical reconstruction of length, version and offset of the prosthesis-allograft construct,

(3) maintaining the bone-soft tissue attachment to the host femur, to provide both mechanical stability and to act as a vascularised graft.

A constrained acetabular liner may be considered in cases of minimal abductor musculature.

\section{Conclusion}

The continued followup and analysis of this technique should be encouraged to refine and develop the management of massive proximal femoral bone loss. This review demonstrates that proximal femoral allografts for revision hip arthroplasty in femoral segmental bone loss do provide a durable solution, with current available evidence reporting a survivorship of $80 \%$. Whilst a range of surgical techniques have been described, this study highlights the following:

(1) high caseload is associated with a lower infection rate,

(2) uncemented distal fixation is associated with a reduced the risk of aseptic loosening or fracture,

(3) if available, using the host femur as an onlay graft enhances hip stability whilst acting as a vascularised graft.

\section{References}

[1] H. R. L. Blackley, A. M. Davis, C. R. Hutchison, and A. E. Gross, "Proximal femoral allografts for reconstruction of bone stock in revision arthroplasty of the hip. A nine to fifteen-year follow-up," Journal of Bone and Joint Surgery, vol. 83, no. 3, pp. 346-354, 2001.

[2] L. S. Lohmander, L. B. Engesæter, P. Herberts, T. Ingvarsson, U. Lucht, and T. J. S. Puolakka, "Standardized incidence rates of total hip replacement for primary hip osteoarthritis in the 5 Nordic countries: similarities and differences," Acta Orthopaedica, vol. 77, no. 5, pp. 733-740, 2006.

[3] S. Kurtz, F. Mowat, K. Ong, N. Chan, E. Lau, and M. Halpern, "Prevalence of primary and revision total hip and knee arthroplasty in the United States from 1990 through 2002," Journal of Bone and Joint Surgery, vol. 87, no. 7, pp. 1487-1497, 2005.

[4] The Swedish hip arthroplasty register, "Annual report 2006," 2011, http://www.jru.orthop.gu.se.

[5] D. J. Berry, "Epidemiology: hip and knee," Orthopedic Clinics of North America, vol. 30, no. 2, pp. 183-190, 1999.

[6] H. Chandler, J. Clark, S. Murphy et al., "Reconstruction of major segmental loss of the proximal femur in revision total hip arthroplasty," Clinical Orthopaedics and Related Research, no. 298, pp. 67-74, 1994.

[7] C. P. Duncan, E. L. Masterson, and B. A. Masri, "Impaction allografting with cement for the management of femoral bone loss," Orthopedic Clinics of North America, vol. 29, no. 2, pp. 297-305, 1998.

[8] B. R. Halliday, H. W. English, A. J. Timperley, G. A. Gie, and R. S. Ling, "Femoral impaction grafting with cement in revision total hip replacement. Evolution of the technique and results," Journal of Bone and Joint Surgery, vol. 85-86, pp. 809-817, 2003.

[9] W. G. Paprosky and R. Aribindi, "Hip replacement: treatment of femoral bone loss using distal bypass fixation," Instructional Course Lectures, vol. 49, pp. 119-130, 2000.

[10] W. G. Paprosky, N. V. Greidanus, and J. Antoniou, "Minimum 10 -year-results of extensively porous-coated stems in revision hip arthroplasty," Clinical Orthopaedics and Related Research, no. 369, pp. 230-242, 1999.

[11] A. L. Malkani, F. H. Sim, and E. Y. S. Chao, "Custom-made segmental femoral replacement prosthesis in revision total hip 
arthroplasty," Orthopedic Clinics of North America, vol. 24, no. 4, pp. 727-733, 1993.

[12] J. Parvizi and F. H. Sim, "Proximal femoral replacements with megaprostheses," Clinical Orthopaedics and Related Research, no. 420, pp. 169-175, 2004.

[13] R. J. Zehr, W. F. Enneking, and M. T. Scarborough, "Allograftprosthesis composite versus megaprosthesis in proximal femoral reconstruction," Clinical Orthopaedics and Related Research, no. 322, pp. 207-223, 1996.

[14] E. R. Henderson, J. S. Groundland, E. Pala et al., "Failure mode classification for tumor endoprostheses: retrospective review of five institutions and a literature review," Journal of Bone and Joint Surgery, vol. 93-95, pp. 418-429, 2011.

[15] B. J. A. Mohit, "Meta-analysis," in Clinical Research for Surgeons, B. J. A. Mohit, Ed., pp. 68-76, Thieme, New York, NY, USA, 2009.

[16] F. Langlais, J. C. Lambotte, P. Collin, and H. Thomazeau, "Long-term results of allograft composite total hip prostheses for tumors," Clinical Orthopaedics and Related Research, no. 414, pp. 197-211, 2003.

[17] F. S. Haddad, M. J. Spangehl, B. A. Masri, D. S. Garbuz, and C. P. Duncan, "Circumferential allograft replacement of the proximal femur: a critical analysis," Clinical Orthopaedics and Related Research, no. 371, pp. 98-107, 2000.

[18] J. C. Zmolek and L. D. Dorr, "Revision total hip arthroplasty: the use of solid allograft," Journal of Arthroplasty, vol. 8, no. 4, pp. 361-370, 1993.

[19] O. Safir, C. F. Kellett, M. Flint, D. Backstein, and A. E. Gross, "Revision of the deficient proximal femur with a proximal femoral allograft," Clinical Orthopaedics and Related Research, vol. 467, no. 1, pp. 206-212, 2009.

[20] L. Vastel, C. T. Lemoine, M. Kerboull, and J. P. Courpied, "Structural allograft and cemented long-stem prosthesis for complex revision hip arthroplasty: use of a trochanteric claw plate improves final hip function," International Orthopaedics, vol. 31, no. 6, pp. 851-857, 2007.

[21] G. C. Babis, V. I. Sakellariou, M. I. O’Connor, A. D. Hanssen, and F. H. Sim, "Proximal femoral allograft-prosthesis composites in revision hip replacement: a 12-year follow-up study," Journal of Bone and Joint Surgery, vol. 92, no. 3, pp. 349-355, 2010.

[22] S. H. Lee, Y. J. Ahn, S. J. Chung, B. K. Kim, and J. H. Hwang, "The use of allograft prosthesis composite for extensive proximal femoral bone deficiencies. A 2- to 9.8-year follow-up study," Journal of Arthroplasty, vol. 24, no. 8, pp. 1241-1248, 2009.

[23] P. J. Roque, H. J. Mankin, and H. Malchau, "Proximal femoral allograft: prognostic indicators," Journal of Arthroplasty, vol. 25, no. 7, pp. 1028-1033, 2010.

[24] D. J. Biau, F. Larousserie, F. Thévenin, S. Piperno-Neumann, and P. Anract, "Results of 32 allograft-prosthesis composite reconstructions of the proximal femur," Clinical Orthopaedics and Related Research, vol. 468, no. 3, pp. 834-845, 2010.

[25] D. Donati, S. Giacomini, E. Gozzi, and M. Mercuri, "Proximal femur reconstruction by an allograft prosthesis composite," Clinical Orthopaedics and Related Research, vol. 394, pp. 192200, 2002.

[26] Y. Farid, P. P. Lin, V. O. Lewis, and A. W. Yasko, "Endoprosthetic and allograft-prosthetic composite reconstruction of the proximal femur for bone neoplasms," Clinical Orthopaedics and Related Research, vol. 442, pp. 223-229, 2006.

[27] N. M. Graham and I. Stockley, "The use of structural proximal femoral allografts in complex revision hip arthroplasty,"
Journal of Bone and Joint Surgery, vol. 86, no. 3, pp. 337-343, 2004.

[28] D. L. Muscolo, G. L. Farfalli, L. A. Aponte-Tinao, and M. A. Ayerza, "Proximal femur allograft-prosthesis with compression plates and a short stem," Clinical Orthopaedics and Related Research, vol. 468, no. 1, pp. 224-230, 2010.

[29] J. W. Wang and C. J. Wang, "Proximal femoral allografts for bone deficiencies in revision hip arthroplasty: a medium-term follow-up study," Journal of Arthroplasty, vol. 19, no. 7, pp. 845-852, 2004.

[30] D. Lakstein, D. J. Backstein, O. Safir, Y. Kosashvili, and A. E. Gross, "Modified trochanteric slide for complex hip arthroplasty. Clinical outcomes and complication rates," Journal of Arthroplasty, vol. 25, no. 3, pp. 363-368, 2010.

[31] D. Lakstein, Y. Kosashvili, D. Backstein, O. Safir, and A. E. Gross, "Trochanteric slide osteotomy on previously osteotomized greater trochanters," Clinical Orthopaedics and Related Research, vol. 468, no. 6, pp. 1630-1634, 2010.

[32] D. Lakstein, Y. Kosashvili, D. Backstein, O. Safir, and A. E. Gross, "Modified extended trochanteric osteotomy with preservation of posterior structures," HIP International, vol. 20, no. 1, pp. 102-108, 2010.

[33] A. E. Gross and C. R. Hutchison, "Proximal femoral allografts for reconstruction of bone stock in revision hip arthroplasty," Orthopedics, vol. 21, no. 9, pp. 999-1001, 1998. 


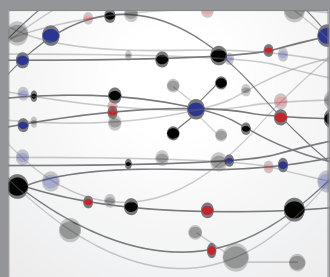

The Scientific World Journal
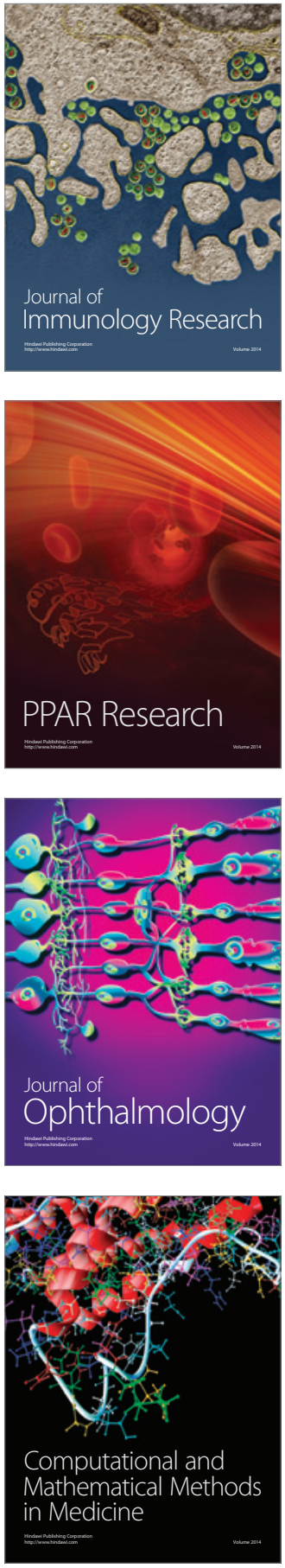

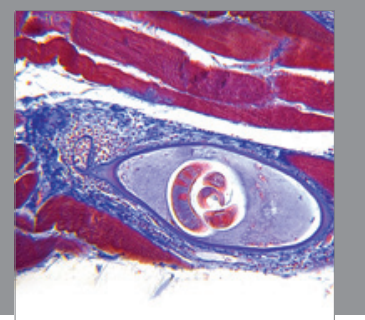

Gastroenterology

Research and Practice
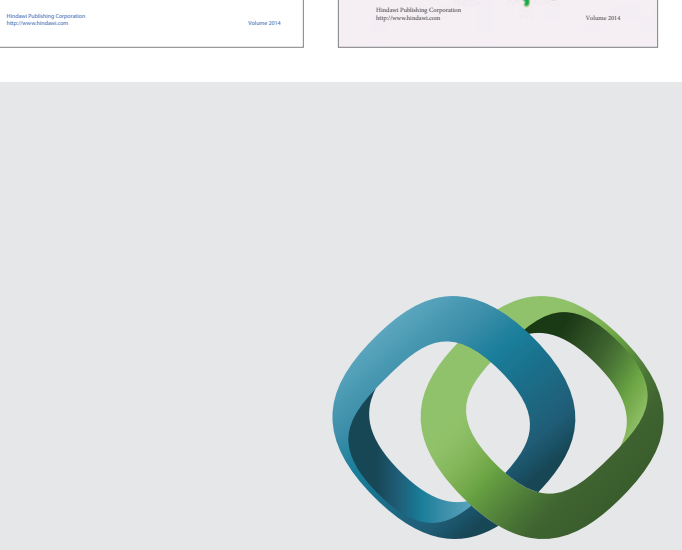

\section{Hindawi}

Submit your manuscripts at

http://www.hindawi.com
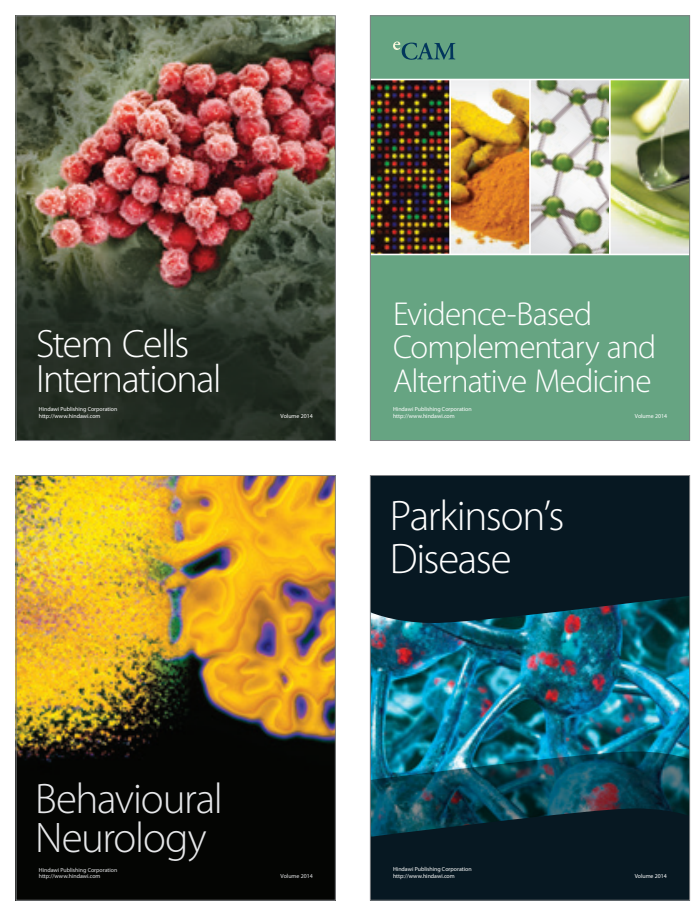

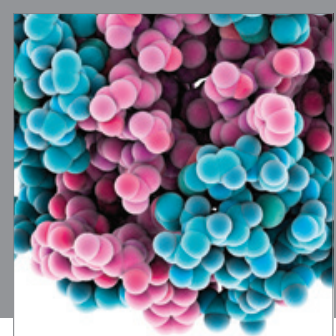

Journal of
Diabetes Research

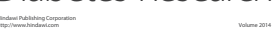

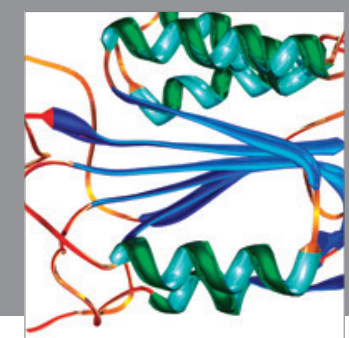

Disease Markers
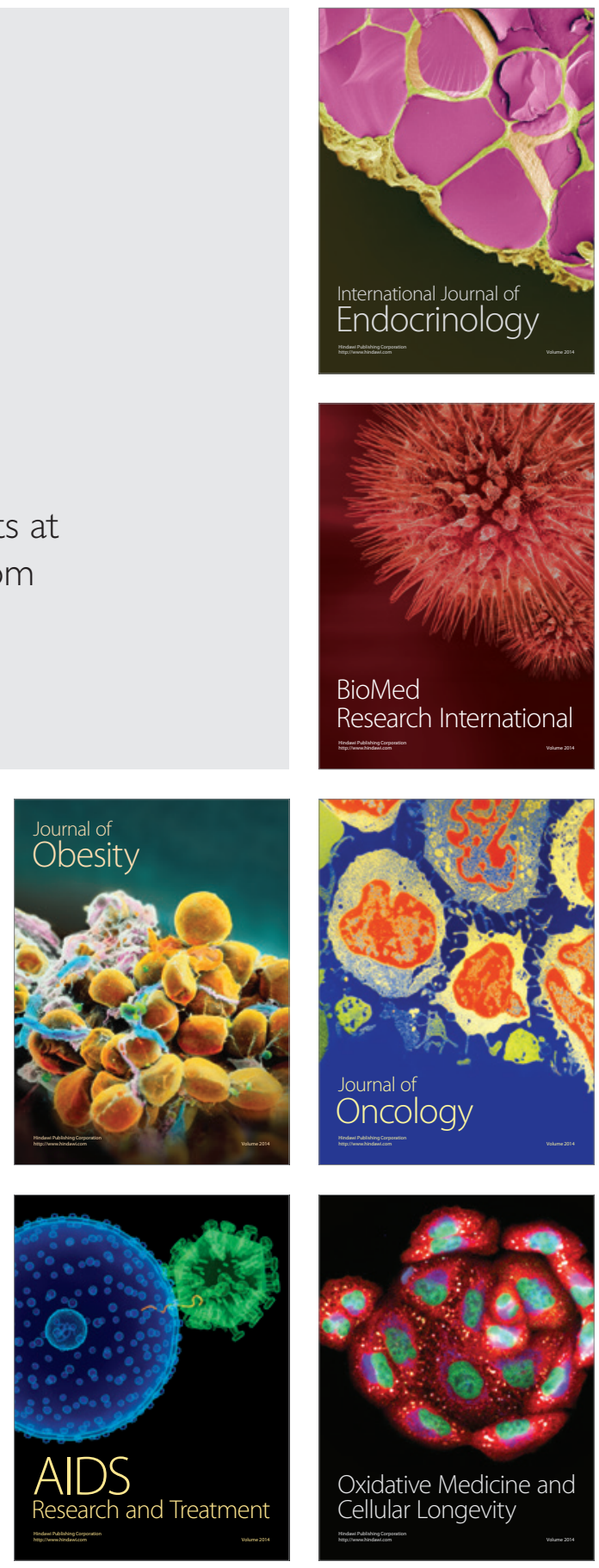